\title{
Variabilidade temporal da evapotranspiração real e da razão entre evapotranspiração real e potencial em Campinas, Estado de São Paulo
}

\author{
Gabriel Constantino Blain (*); Regina Clélia de Matos Pires \\ Instituto Agronômico (IAC), Centro de Pesquisa e desenvolvimento de Ecofisiologia e Biofísica, Caixa Postal 28, 13012-970 \\ Campinas (SP). \\ (*) Autor correspondente: gabriel@iac.sp.gov.br
}

Recebido: 24/mar./2010; Aceito: 31/ago./2010.

\begin{abstract}
Resumo
O objetivo do trabalho foi caracterizar a variabilidade temporal de séries decendiais de evapotranspiração real (ETR) e da razão entre a evapotranspiração real e potencial (ETR/ETP) na localidade de Campinas, Estado de São Paulo, no período de 1948-2008, considerando três valores de capacidade de água disponível (CAD): 50, 100 e 150 mm. Utilizando a função autocorrelação (acf) verificou-se presença de significativa persistência temporal nos valores de ETR e de ETR/ETP. A aplicação da acf também permitiu inferir que essa persistência eleva-se com o aumento do valor da CAD. A análise espectral indicou queda nos picos de potência dessas duas variáveis a partir de 1976. A influência do El Nino/oscilação sul nas séries de ETR e ETR/ETP não é conclusiva. A aplicação do método Sazonal de Mann-Kendall indicou, em janeiro, maio, julho, setembro, novembro e dezembro, tendências de elevação na ETR. A ETR/ETP foi pouco sensível as elevações temporais observadas nas séries de ETP.
\end{abstract}

Palavras-chave: Mudança climática, variabilidade temporal, tendências.

\section{Temporal variability of the actual evapotranspiration and the ratio between actual and potential evapotranspiration in Campinas, State of São Paulo, Brazil}

\begin{abstract}
The aim of the work was to characterize the temporal variability of ten-day evapotranspiration series, considering the actual evapotranspiration (ETa) and the ratio between actual and potential evapotranspiration (ETa/ETP). Data between 1948 and 2008 were collected with a weather station in Campinas, State of São Paulo, Brazil. Evapotranspiration calculation considered three values of soil water availability (SWA): 50, 100, and $150 \mathrm{~mm}$. Based on autocorrelation function, it was detected a significant level of temporal persistence on ETa and ETa/ETP series. The acf also has indicated that this temporal persistence tends to increase due to high SWA values. The spectral analysis has shown a decreasing power of these series after 1976. The El Niño/southern oscillation influence on ETa and ETa/ETP temporal variability was not conclusive. The Seasonal Mann-Kendall test has shown increasing trends on ETa series in the months of January, May, July, September, November and December. The ETa/ETP series was little affected by the increasing trends detected on ETP series.
\end{abstract}

Key words: Climate change, temporal variability, trends.

\section{INTRODUÇÃO}

Devido ao caráter probabilístico das séries temporais oriundas da observação de qualquer parâmetro agrometeorológico, um dos maiores desafios da estatística climatológica é estabelecer algoritmos apropriados para distinguir flutuaçóes controladas por variaçóes estocásticas de reais tendências climáticas. Nesse aspecto, a limitação na duração das séries meteorológicas pode tanto resultar na falsa indicação de mudanças climáticas, quanto mascarar reais alteraçóes no clima regional (Омм, 1996).
Considerando que o termo mudança climática é definido pelo IPCC (2007) por alteraçóes nos padrōes climáticos, que podem ser identificadas por meio de variaçóes persistentes por longos períodos (da ordem de décadas), autores como Richards (1993); Frei e SChar (2000); Blain (2009); Blain et al. (2009a,b) e indicam algoritmos apropriados a detecção dessas variaçôes persistentes em séries meteorológicas.

Sob o aspecto agrometeorológico uma das preocupaçóes relativas aos impactos do aquecimento global é a diminuição da disponibilidade hídrica às culturas decorrente de alteraçôes na variabilidade da precipi- 
tação pluvial (PRE) e da temperatura do ar (T). Essas alteraçôes, por sua vez, podem diminuir a produçáo de alimentos devido à redução das trocas gasosas no sistema planta-atmosfera proporcionada pela ocorrência de estresse hídrico. Dessa forma, a investigação de possíveis tendências em séries temporais de parâmetros agrometeorológicos, relacionados ao atendimento hídrico das culturas, torna-se relevante.

Considerando que trabalhos como os de Moraes (1998), Ortolani et al. (1998), Camargo e Hubbard (1999), Piccini et al. (1999), Camargo et al. (2007) e o modelo "FAO" (Doorenbos e KasSAM, 1994) relacionam a redução de produtividade agrícola à razão entre a evapotranspiração real (ETR) e a potencial (ou de referência; ETP), a caracterização da variabilidade temporal de séries constituídas por esse parâmetro (ETR/ETP), tornase uma opção natural aos estudos dos possíveis impactos agrícolas dos cenários de clima futuro.

Analisando a frequência de ocorrência de classes de valores da razão ETR/ETP em Campinas (SP), BLAIN et al. (2009c) afirmam não haver marcantes alteraçóes nas formas e escalas das funções densidade de probabilidade, associadas aos anos de 1948 a 1976 e 1977 a 2006, relativas a esse parâmetro agrometeorológico. Contudo, conforme Ortolani e Camargo (1987), o clima da localidade de Campinas é caracterizado por um período, outubro a março, em que a precipitação é consideravelmente superior à evapotranspiração potencial. Nesse contexto, é possível inferir que elevaçôes temporais nos dados de ETP relativos, por exemplo, ao primeiro decêndio de janeiro, resultarão, em princípio e desde que não haja redução nos totais de PRE, no aumento do valor da ETR; mantendo inalterado o quociente ETR/ETP. Consequentemente, a análise de tendências climáticas baseadas apenas neste último parâmetro do balanço hídrico pode não revelar importantes alteraçóes na variabilidade climática da região de Campinas.

Dessa forma, o objetivo do trabalho é caracterizar a variabilidade temporal de séries decendiais de ETR e ETR/ETP, na localidade de Campinas, entre 1948 e 2008, investigando o grau de persistência temporal, picos de variância e possíveis tendências de ordem climática presentes nos dados.

\section{MATERIAL E MÉTODOS}

Foram utilizados dados decendiais de totais de precipitação pluvial e temperatura média do ar, pertencentes ao Instituto Agronômico (IAC/SAA-SP; Campinas 1948 a 2008; 22054'S; 4705'W; 669m). O balanço hídrico sequencial, que gerou os valores decendiais de ETR e de ETR/ETP, foi calculado conforme ThornthwaIte e Matter (1955) com a evapotranspiração potencial estimada com base em Thonthwarte (1948). Utilizou-se o software desenvolvido por Rolim et al. (1998), para a estimativa da ETR e da ETP, adotando-se três valores de capacidade de água disponível (CAD) 50, 100 e 150 mm, escolhidos de forma que representem diferentes combinaçôes de armazenamento de água no solo e profundidades de sistema radicular de culturas. É importante ressaltar que a robustez de uma análise estatística voltada ao estudo de mudanças climáticas está fortemente relacionada ao comprimento temporal das séries (agro)meteorológicas. Nesse contexto, o período de 61 anos, adotado no presente estudo, impossibilitou a adoçáo do método de Penmam-Monteith, padrão FAO (Allen et al., 1998), tendo em vista que apenas dados de T e PRE são disponíveis no referido período.

Empregou-se o método de Wald-Wofowitz (run) conforme descrito em Morettin e Toloi (2006) e a função autocorrelaçáo (acf) utilizada em WiLks (2006), para a verificação da correlação serial presente nas séries temporais de ETR e ETR/ETP. Os coeficientes da acf foram estimados até o deslocamento (lag) 36 que em séries decendiais corresponde ao período de um ano. Esse deslocamento, ou número de lags, foi arbitrariamente escolhido, respeitando-se, entretanto, o limite $\mathrm{N} / 4$, em que $\mathrm{N}$ é o número de dados da série. A hipótese de nulidade $\left(\mathrm{H}_{0}\right)$ associada ao teste run e a acf indicam que a série sob investigaçáo pode ser considerada livre de persistência temporal, sendo verdadeira para $\mathrm{p}>0,05$.

A periodicidade dos picos de variância das séries foi investigada utilizando-se a análise de ondaleta $(\mathrm{AO})$, conforme descrita, em Torrence e Compo (1998), Reboita et al. (2006), Kayano e Blain (2007) e Blain (2009). Esta análise foi realizada utilizando o algoritmo elaborado por C. Torrence disponível em http://paos.colorado.edu/ research/wavelets, na linguagem utilizada pelo software Matlab. O nível de significância da $\mathrm{AO}$ adotado na estimaçáo do espectro da ondaleta (Wavelet power spectrum; WPS) foi calculado segundo Torrence e COMPO (1998). A AO foi aplicada aos resíduos decendiais da ETR e da ETR/ETP. Este último foi obtido subtraindo-se de cada valor de ETR ou ETR/ETP a respectiva média aritmética decendial.

De acordo com Grinsted et al. (2004), é interessante interpretar a análise cruzada de ondaleta como o coeficiente de correlação de Pearson, localizado no domínio tempo-frequência. A descriçâo completa desse método é dada em Grinsted et al. (2004). A análise cruzada de ondaleta foi aplicada considerando-se as séries de ETR e ETR/ETP, para os três valores de CAD, e do Índice de Oscilação Sul (IOS). O IOS é um índice de pressão atmosférica utilizado por autores como Torrence e Compo (1998) e Reboita et al. (2006) para determinar as diferentes fases do fenômeno Oceânico-Atmosférico El Niño-Oscilação Sul (ENOS). Segundo Reboita et al. (2006), a Oscilação Sul foi pioneiramente observada por Sir Gilbert Walker em 1924 ao notar a alternância das condiçôes da pressão 
atmosférica ao nível médio do mar (PNM) entre as localidades de Darwin, Austrália (12 oS e 130 E) e do Tahiti (17 oS e $\left.150^{\circ} \mathrm{W}\right)$. O IOS é uma medida da diferença de PNM entre essas duas regiốes. Valores negativos do IOS caracterizam eventos de El Nińo, ao passo que valores positivos caracterizam episódios de La Nińa.

O teste de Sazonal de Mann-Kendall (Mann, 1945; Kendall e Stuart, 1967; Hirsch et al., 1982 e Hirsch e SLACK, 1984) foi utilizado a fim de verificar possíveis tendências, de elevação ou queda, presente nos dados. Valores positivos desse método náo paramétrico indicam que na série sob análise há tendência de elevação em seus dados. Valores negativos do SMK indicam tendência de queda. $\mathrm{O}$ teste não paramétrico de Pettitt (Petтitt, 1979), utilizado por autores como Victoria et al. (1997) e Mortatti et al. (2004) para indicar o ponto inicial de alteração em uma série temporal (changing point), foi aplicado nas séries decendiais de ETR e ETR/ETP em que foram verificados valores do SMK com significância estatística $\mathrm{p}<0,10$. Os testes $S M K$ e Pettitt foram também aplicados às séries decendiais de ETP e de PRE, a fim investigar possíveis tendências nas séries temporais dessas últimas duas variáveis.

\section{RESULTADOS E DISCUSSÃO}

Ao contrário do que ocorre com a temperatura mínima em Campinas (BLAin et al. 2009a), a observaçáo da figura 1 não indica marcantes tendências de elevação ou queda, nos valores de ETR e ETR/ETP. Assim, a aplicação de testes de hipóteses voltados à detecção de tendências climáticas, tais como o SMK e o de Pettitt, torna-se necessária. É também possível verificar que apesar de nenhuma periodicidade ou ciclicidade ser evidente nos resíduos ilustrados na figura 1, nota-se que os maiores valores residuais, tanto da ETR quando da ETR/ETP, ocorrem antes da década de 1980. Nesse aspecto, espera-se que a análise de ondaleta releve queda de energia nos picos de variância relativos aos últimos anos dessas duas séries.

\section{Persistência temporal}

A ETR das culturas é igual à ETP em condiçôes ideais de disponibilidade de água no solo. Por outro lado, à medida que a umidade do solo diminui e que não é mais possível manter a transpiração das plantas nas condiçôes potenciais ou máxima, a ETR assume valores menores do que a ETP estabelecendo-se condiçóes de deficiência hídrica (Pereira et al., 2002). O resultado desse processo, sob o enfoque estatístico, é a presença da persistência temporal ou autocorrelação nas séries de ETR e ETR/ ETP, uma vez que o valor desses parâmetros agrometeorológicos relativos a um dado decêndio estão, entre ou- tros, condicionados pelas condiçóes prévias de umidade do solo e, consequentemente, pelos valores de ETR e/ou ETR/ETP observados em decêndios anteriores. Dessa forma, métodos estatísticos que assumem independência dos dados da amostra não podem ser aplicados às séries do estudo. $\mathrm{O}$ uso de funções densidade de probabilidade, associadas às distribuiçóes paramétricas, para a estimação das probabilidades de ocorrência dos dados decendiais de ETR e ETR/ETP, deve ser considerado com ressalvas.

Corroborando, evidentemente, essa premissa, $\mathrm{H}_{0}$ associada ao teste de Wald - Wofowitz não pode ser aceita, uma vez que seus valores finais ultrapassaram, para os dois parâmetros agrometeorelógicos, o limite de significância adotado $(\mathrm{p} \leq 0,05)$. Essa última característica também indica a necessidade de empregar a adaptação para séries de dados com persistência temporal, proposta por Hirsch et al. (1982) e Hirsch e SLACK (1984) no método original de Mann-Kendall.

$\mathrm{Na}$ figura 2 verifica-se que os coeficientes da acf, aplicada à série de ETR, permaneceram acima do limite superior de significância adotado até o lag (decêndio) oito, para CAD $=50 \mathrm{~mm}$; e 13 para CAD 100 e 150 $\mathrm{mm}$. Para a razão ETR/ETP verifica-se coeficientes significativos até o sexto lag, para CAD $=50 \mathrm{~mm}, 12$ e 13 para CAD 100 e $150 \mathrm{~mm}$ respectivamente. Essa elevaçáo na persistência temporal das séries de ETR e ETR/ ETP, causada pelo aumento do valor da CAD, pode ser vista como descrição matemática de que o aprofundamento do sistema radicular de uma cultura, aliado às condiçôes favoráveis de armazenamento de água no solo favorecem o desenvolvimento das plantas com melhor aproveitamento das precipitaçóes naturais. Nesse aspecto, é também interessante ressaltar que na figura 1 é visível a elevação da estabilidade na variabilidade temporal dos valores de ETR e, especialmente, ETR/ ETP com o aumento do valor da CAD. Desta forma, verifica-se que a variação da memória dessas séries tende a ser diretamente relacionada à variação do valor da CAD. Essa inferência é coerente com a elevação do grau de persistência temporal, também consequente do aumento do valor da CAD, descrita na figura 2 e com os valores das variâncias das séries de ETR/ETP para CAD de 50, 100 e $150 \mathrm{~mm}\left(0,09 \mathrm{~mm}^{2}, 0,06 \mathrm{~mm}^{2}\right.$ e, $0,04 \mathrm{~mm}^{2}$ respectivamente). As variâncias das séries de ETR, para esses três valores de CAD são, respectivamente, $168 \mathrm{~mm}^{2}, 145 \mathrm{~mm}^{2}$ e $132 \mathrm{~mm}^{2}$.

Os resultados do teste Wald - Wofowitz e as informaçôes descritas na figura 2 indicam também que o espectro de fundo utilizado para determinar o nível de significância do espectro de ondaleta deve ser o denominado de ruído vermelho. Este último, conforme descrito por autores como Torrence e Compo (1998), é caracterizado pelo aumento da energia (increasing power) com a queda da frequência. 

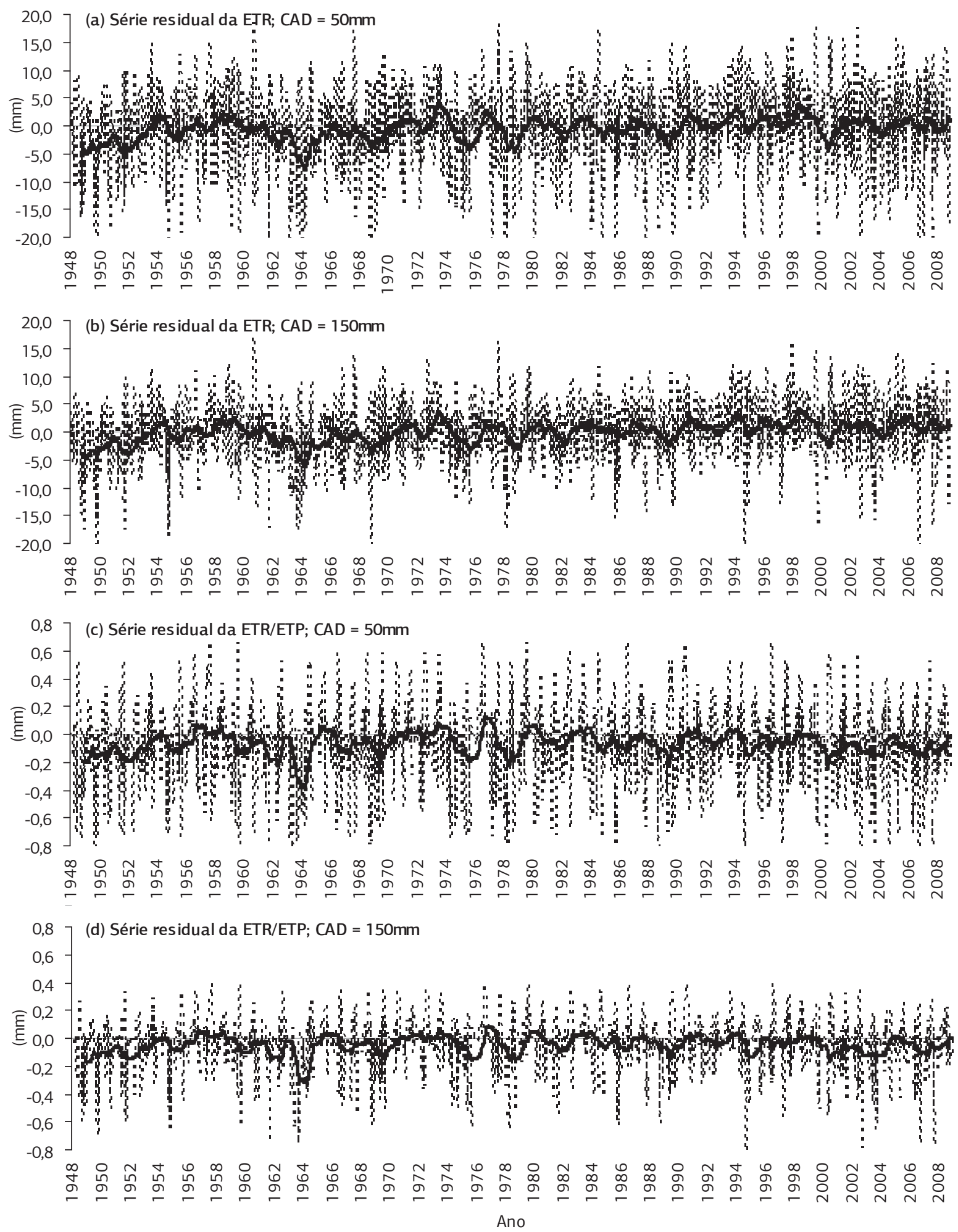

Figura 1. Série residual da evapotranspiração real (ETR) e da razão entre evapotranspiração real e potencial (ETR/ETP) em Campinas (SP), para dois valores de capacidade de água disponível (CAD). A linha pontilhada representa os valores de cada decêndio. A linha cheia representa a média móvel com período arbitrário de 36 decêndios. O resíduo foi obtido após remoção das médias decendiais.

\section{Análise espectral}

$\mathrm{Na}$ figura 3 a, c, e é possível verificar picos de variância, com escala próxima a um ano, no início das décadas de 1950, 1960 e fim da de 1970. Nota-se também elevada concentração de energia, com escala próxima a três anos, entre o início da década de 1970 até início da de 1980. De forma geral, e coerente com a figura 1, nota-se queda nos picos de variância das séries de ETR a partir do fim da década de 1970 . 

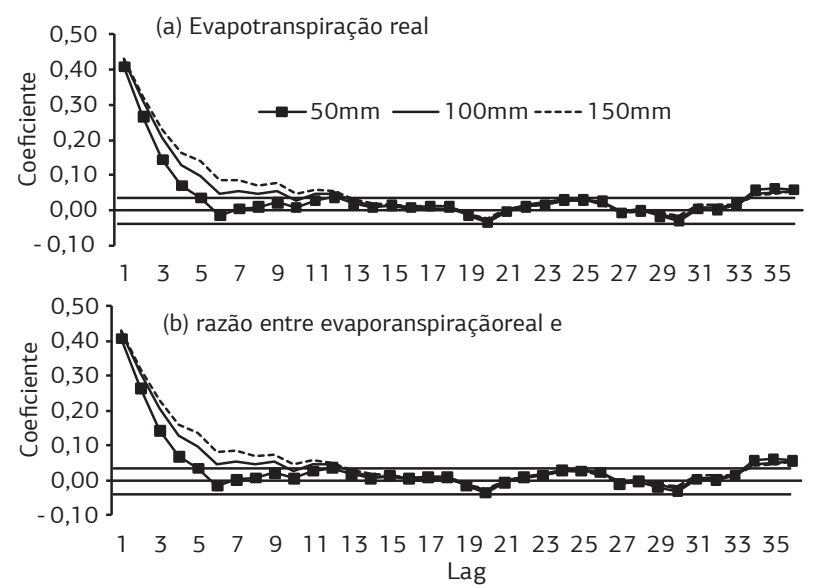

Figura 2. Coeficientes da função autocorrelaçấo aplicada (a) às séries decendiais de evapotranspiração real e (b) à razão entre a evapotranspiração real e potencial, considerando três valores de capacidade de água disponível (CAD; 50, 100 e $150 \mathrm{~mm}$ ) na localidade de Campinas (SP), de 1948 a 2008. As linhas horizontais representam os limites do ruído branco, além dos quais a série não pode ser considerada livre de persistência temporal.
Para a potência global da ondaleta (Figura 3b,d,f), observa-se um pico significativo de variância, com escala temporal entre três e quatro anos. Nesse contexto, é interessante ressaltar que autores como TORRENCE e COMPO (1998), ao aplicarem o método da ondaleta em sinais temporais do ENOS, indicam que a maior parte da energia ("most of the power") resultante desta análise, concentra-se na escala de dois a oito anos (esta última é usualmente denominada de banda do ENSO).

Na potência local da ondaleta relativa às séries de ETR/ ETP (Figura 4a,c,e), verifica-se até o fim da década de 1970 considerável frequência de picos de variância com escala próxima à anual. A partir de 1980, há um deslocamento desse sinal espectral para uma escala ligeiramente inferior, mais próxima de 0,5 ano. Nas décadas de 1960, 1970 e 1980 há variâncias significativas com escalas aproximadas de 1 a 3 e 2 a 3 anos respectivamente. Assim como para o sinal da ETR, há queda na energia da ondaleta após o fim da década de 1970 nas séries de ETR/ETP. Em outras palavras, a ETR e a ETR/ETP permaneceram mais próximas (a) WPS - CAD = $50 \mathrm{~mm}$

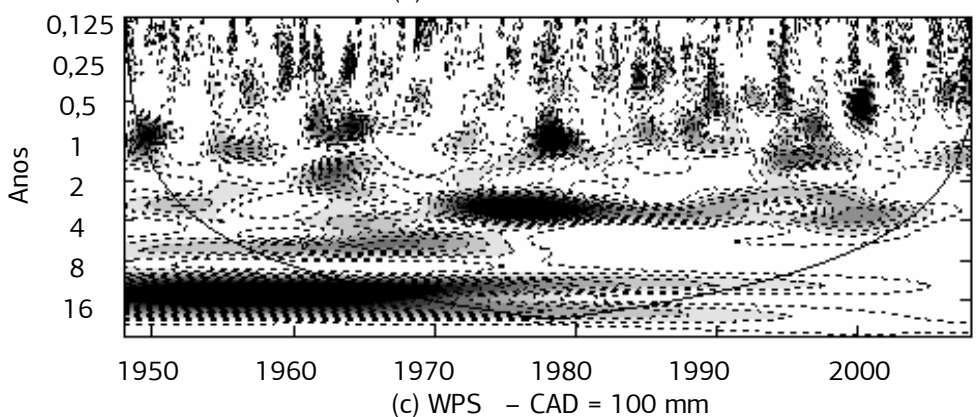

(c) WPS - CAD $=100 \mathrm{~mm}$
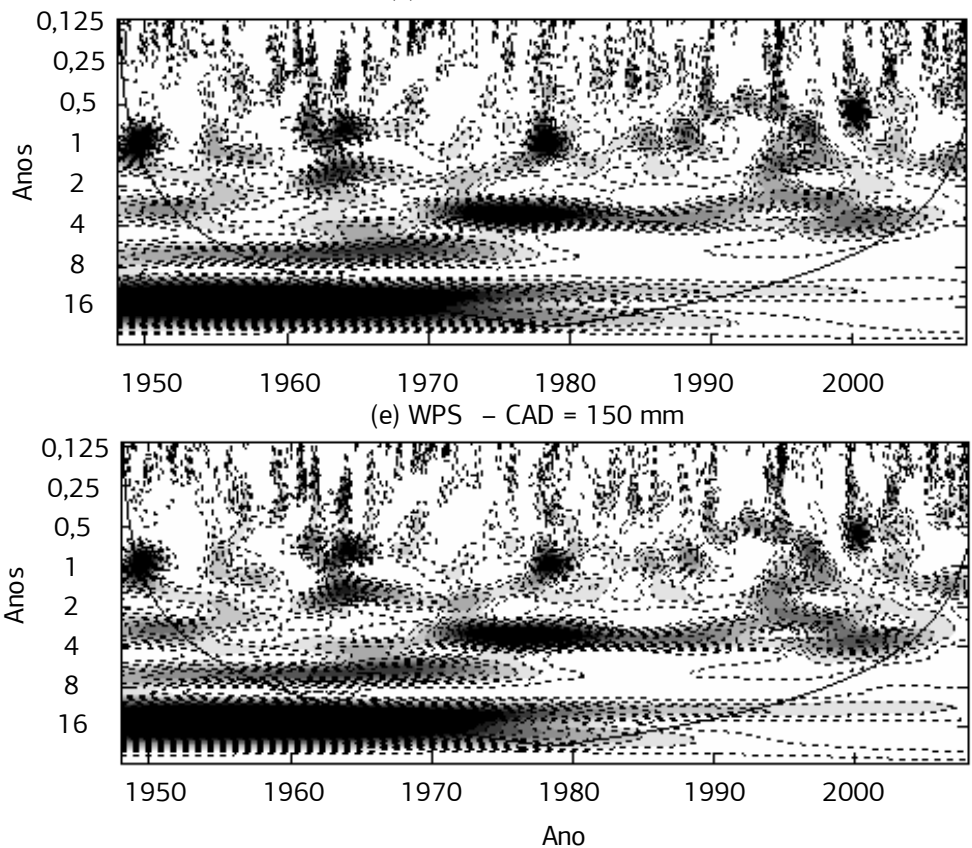

(b) GWP
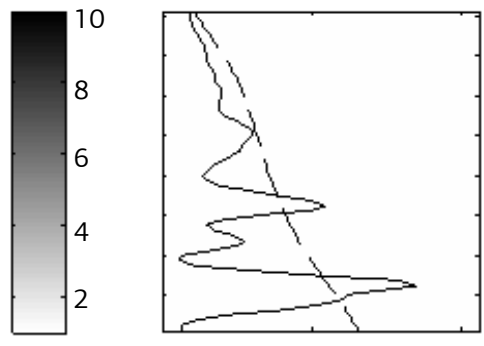

200

(d) GWP
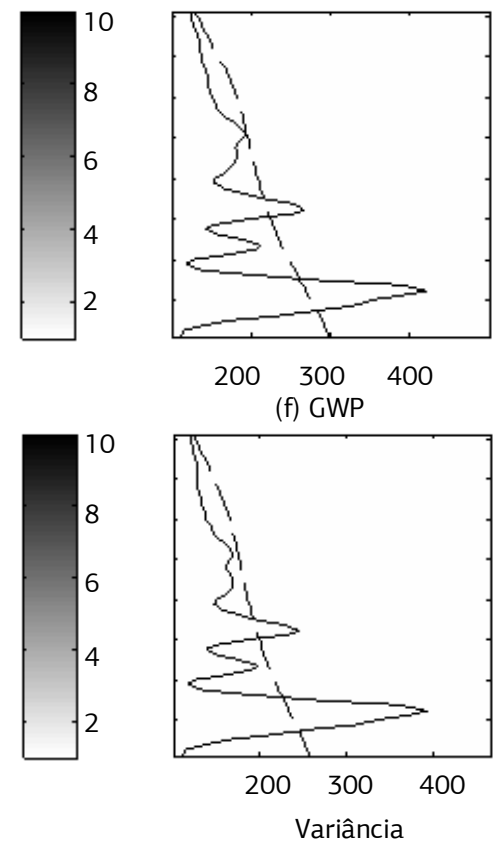

Figura 3. Espectro de energia local da ondaleta (WPS) para a evapotranspiração real residual decendial de Campinas (SP), para três valores de capacidade de água disponível (CAD). Contornos tracejados correspondem a valores de variância normalizados variando de 1 a 10 . Contornos sombreados englobam áreas com variâncias significativas ao nível de $95 \%$ de confiança. Espectro global de ondaleta (GWP) também é ilustrado. A linha em forma de U representa a área denominada "cone de influência" em que o efeito de borda é relevante. 
(a) WPS - CAD $=50 \mathrm{~mm}$

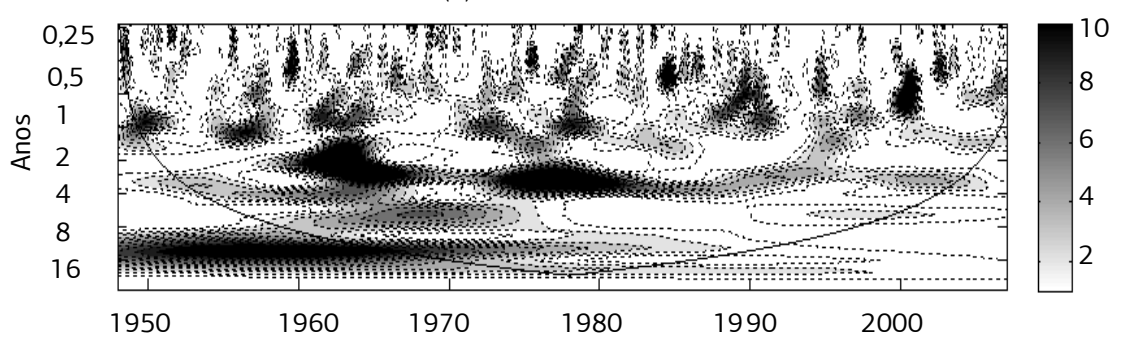

(c) WPS $-C A D=100 \mathrm{~mm}$

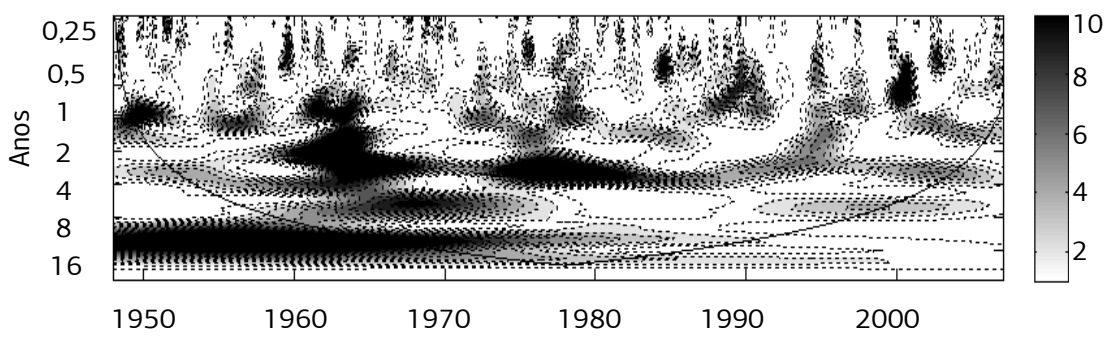

(e) WPS - CAD $=150 \mathrm{~mm}$

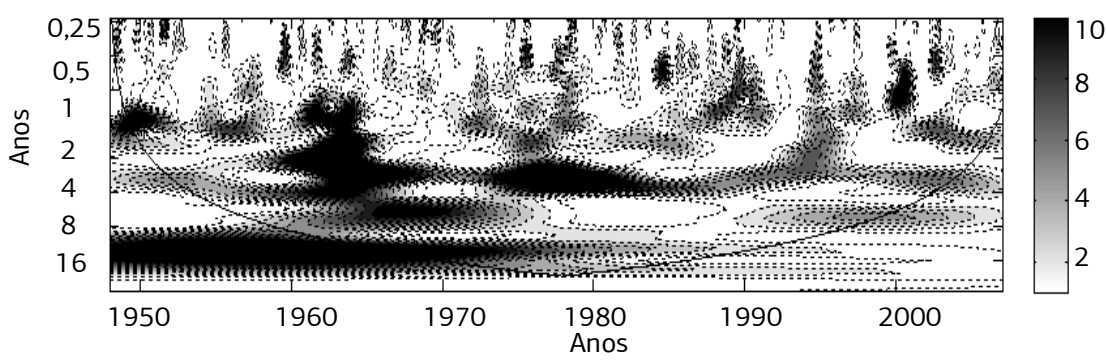

(b) GWP

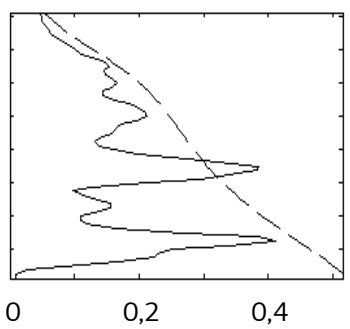

(d) GWP

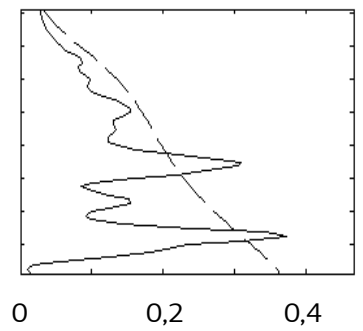

(f) GWP

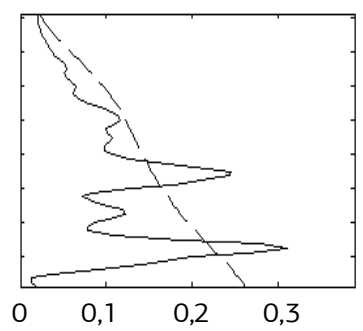

Figura 4. Espectro de energia local da ondaleta (WPS) para a razão entre a evapotranspiração real e potencial residual decendial de Campinas (SP), para três valores de capacidade de água disponível (CAD). Contornos tracejados correspondem a valores de variância normalizados variando de 1 a 10 . Contornos sombreados englobam áreas com variâncias significativas ao nível de $95 \%$ de confiança. Espectro global de ondaleta (GWP) também é ilustrado. A linha em forma de U representa a área denominada "cone de influência" em que o efeito de borda é relevante.

de suas respectivas médias decendiais a partir da década de 1980. A potência global da ondaleta (Figura 4b,d,f) proporciona picos significativos de variância com de escalas 2 a 4 anos, para os três valores de CAD.

Esses picos de variância observados na potência global da ondaleta dos sinais da ETR/ETP e, especialmente, da ETR dão margem à hipótese de possíveis influências do fenômeno ENOS na variabilidade temporal dos dois parâmetros agrometeorológicos sob investigação. Contudo, é importante ressaltar que Blain (2009) e Blain et al. (2009b) indicam que a influência desse fenômeno oceânico-atmosférico, nas condiçóes climáticas da região de Campinas não é significativa. Sob esse aspecto, RaO e HADA (1994) também apontam que a análise da influência do ENOS na Região Sudeste do Brasil, não é conclusiva.

Por meio da aplicação da análise cruzada da ondaleta, foi possível comprovar a dificuldade em estabelecer relação conclusiva entre a variabilidade temporal do ENOS e as séries de ETR e ETR/ETP utilizadas. Coerências significativas, na escala de 2 a 8 anos ocorreram, para os três valores de $\mathrm{CAD}$, em apenas alguns curtos intervalos ao longo do período analisado, conforme ilustrado na figura 5 para $\mathrm{CAD}=50 \mathrm{~mm}$. Considerando todo o segmento temporal (1948 a 2008; nas diversas escalas de análise) não foi possível estabelecer um padrão comum do ângulo de fase entre as três séries analisadas e o sinal ENOS. Nesse ponto, é importante ressaltar que dois mecanismos ou processos físicos correlacionados, ao serem submetidos à análise cruzada de ondaleta, tendem a ter ângulos de fase constantes em diversas escalas temporais (GRINSTED et al., 2004). Essa indicação de Grinsted et al. (2004) não pôde ser observada, no presente estudo, para nenhum dos valores de CAD adotados. Assim, torna-se possível inferir que a influência do ENOS na variabilidade temporal das séries do estudo não é conclusiva.

As análises espectrais anteriormente descritas referemse às escalas intranual e interanual. Contudo, verifica-se que o pico de variância mais significativo observado na potencia global da ondaleta, relativo à ETR e à ETR/ ETP, ocorre na escala temporal de 10 a 16 anos. Essa característica indica que, entre os anos de 1948 a 2008, a 
variabilidade dominante nessas séries é de escala interdecadal. Ainda para essa escala temporal, nas figuras $3 a, c, e, e$ $4 a, c, e$, observa-se um pico de energia espectral concentrado desde o início da série até meados da década de 1970. Embora parte dessa energia esteja situada fora da denominada área de influência de cone (regiáo em que o efeito de borda é importante) é oportuno destacar que ZHANG et al. (1997; 1998), Mantua et al. (1997) descrevem uma forma de oscilação na temperatura da superfície do Oceano Pacífico similar ao ENOS, ocorrendo, entretanto, em escala multidecadal. A esse fenômeno é atribuído o nome de Oscilação Decadal do Pacífico (ODP). Mantua et al. (1997) define os períodos de 1900 a 1924 e 1947 a 1976 como fases frias da ODP, ao passo que os períodos de 1925 a 1946 e 1977 a 1995 (fim da série utilizada por esses autores) são definidos como fases quentes dessa oscilação. Andreoli e Kayano (2005) e Kayano e Andreoli (2007) demonstraram, para a América do Sul, que quando o ENOS e a PDO estáo na mesma fase, essas oscilaçóes agem de maneira construtiva nos picos de variância do sinal temporal da PRE. Quando esses fenômenos estão na fase oposta, essa influência nos picos de potência do sinal espectral torna-se destrutiva.

Neste estudo, a concomitância entre o pico de potência espectral, de escala interdecadal, e a fase fria da ODP de 1947 a 1976, aliada à queda de energia observada na fase quente da ODP (subsequente a 1976), demonstram que as anomalias nos sinais temporais da ETR e da ETR/ ETP foram mais acentuadas ao longo da fase fria dessa oscilação na temperatura da superfície do Oceano Pacífico do que durante a fase quente. Essa última indicação fornece suporte estatístico para futuras investigaçôes que analisem as influências da PDO na variabilidade temporal da ETR e da ETR/ETP nas demais localidades da Regiáo Sudeste do Brasil.

\section{Tendências climáticas}

De forma geral, verifica-se pela tabela 1 , que nas séries de ETR há tendência de elevação em seus valores. Desse modo, o valor de significância $\mathrm{p}<0,05$ foi atingido apenas nos casos em que o SMK revelou valor positivo. Essa tendência de elevação ocorreu no segundo decêndio de janeiro, julho, setembro e dezembro; no terceiro decêndio de maio e dezembro e no primeiro decêndio de novembro. Para maio e julho, inseridos na estação seca da localidade de Campinas, essa alteração ocorreu a partir do fim da década de 1960. As demais alteraçôes ocorrem nos meses de primavera e verão, com início predominante na década de 1980.

Como esperado, a razão ETR/ETP da localidade de Campinas foi menos sensível a tendências de ordem climática, conforme ilustrado na tabela 2. O valor $\mathrm{p}<0,05$ de significância foi atingido apenas no segundo decêndio de maio, para as CAD 50 e $100 \mathrm{~mm}$, indicando elevação (a) ETR

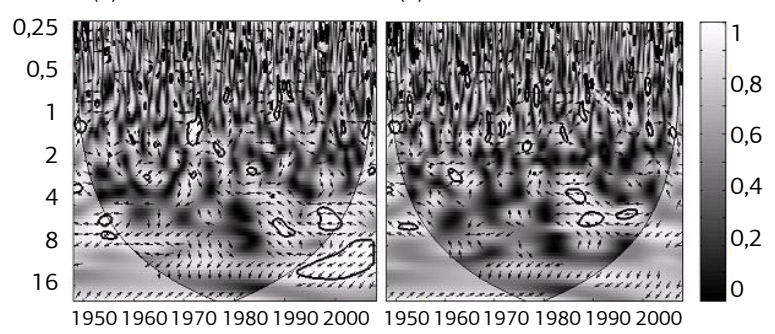

Figura 5. Coerência e diferença de fase (indicadas por setas) do Índice de Oscilação Sul (IOS) e a evapotranspiração real (ETR) decendial (a) para $50 \mathrm{~mm}$ de capacidade de água disponível, na localidade de Campinas (SP). Linhas contínuas de cor preta indicam coerência significativa ao nível de 5\%. Apenas as setas correspondentes à coerência superior a 0,8 foram mostradas. A convenção das setas é a seguinte: horizontais para a direita indicam que as duas séries estão em fase; horizontais para esquerda indicam que as duas séries estão fora de fase; verticais para baixo indicam que a série de evapotranspiração real está $90^{\circ}$ atrasada em relação à do IOS; verticais para cima indicam que a série de evapotranspiração real está a $90^{\circ}$ graus adiantada em relação à do IOS. (b) mesma análise para a razão entre a evapotranspiração real e potencial (ETR/ ETP). A linha em forma de U representa a área denominada "cone de influência” em que o efeito de borda é relevante

nos valores desse parâmetro agrometeorológico. O limite $\mathrm{p}<0,10$ de significância foi alcançado no primeiro decêndio de julho, apenas para CAD $50 \mathrm{~mm}$, e no primeiro e segundo decêndio de setembro e outubro respectivamente. Entre novembro e janeiro e, especialmente, entre maio e setembro, há predominância de casos positivos do SMK. Em outubro e entre fevereiro e abril, valores negativos do SMK são mais frequentes. As análises ilustradas na Tabela 2 são coerentes com as observaçóes de BLAIN et al. (2009c) que, apesar de não indicar a significância estatística de seus resultados, não detectaram marcantes alteraçôes nas probabilidades de ocorrência dos valores da razão ETR/ETP na série de Campinas. No entanto, as tendências observadas nas séries de ETR e de ETR/ ETP em maio corroboram as indicaçôes de BLAIN (2009) e BLAIN et al. (2009b) que afirmam haver significativos indícios de elevação no regime de precipitação pluvial relativa ao referido mês na localidade de Campinas.

Considerando que a variabilidade temporal da ETR e da ETR/ETP está fundamentalmente relacionada aos dados de temperatura média do ar e de precipitação pluvial, torna-se relevante investigar se os valores significativos das tabelas 1 e 2 estão relacionados às tendências de elevação na evapotranspiração potencial, na precipitação pluvial ou em ambos.

Observando a tabela 3, torna-se clara a dificuldade em estabelecer um único nível de significância apropriado à investigação do tema mudança climática. No segundo decêndio de julho, setembro e dezembro e no primeiro decêndio de novembro, os valores do teste SMK, relativos às séries de ETP e PRE não atingiram o nível de 5\% de significância. Contudo, essas tendências (frequentemen- 
Tabela 1. Testes Sazonal de Mann-Kendall (SMK) aplicados às séries decendiais (D) de evapotranspiração real, para três valores de capacidade de água disponível (CAD), na localidade de Campinas (SP), de 1948 a 2008. As significâncias (p) associadas ao teste SMK e o ano de início das tendências climáticas, associadas a $\mathrm{p} \leq 0,10$, são também apresentados.

\begin{tabular}{|c|c|c|c|c|c|c|c|}
\hline \multirow{2}{*}{ Mês } & \multirow{2}{*}{ D } & \multicolumn{2}{|c|}{$C A D=50 \mathrm{~mm}$} & \multicolumn{2}{|c|}{$C A D=100 \mathrm{~mm}$} & \multicolumn{2}{|c|}{$C A D=150 \mathrm{~mm}$} \\
\hline & & SMK & p & SMK & p & SMK & p \\
\hline \multirow{3}{*}{ jan } & 1 & 0,92 & 0,36 & 0,52 & 0,60 & 0,12 & 0,90 \\
\hline & 2 & 1,56 & 0,12 & 2,09 & $0,04(1982)$ & 2,24 & $0,03(1982)$ \\
\hline & 3 & 1,43 & 0,15 & 1,31 & 0,19 & 1,13 & 0,26 \\
\hline \multirow{3}{*}{ fev } & 4 & 1,56 & 0,12 & 1,72 & 0,08 & 1,69 & 0,09 \\
\hline & 5 & 0,66 & 0,51 & 0,66 & 0,51 & 0,61 & 0,54 \\
\hline & 6 & 1,51 & 0,13 & 1,89 & 0,06 (1979) & 1,86 & 0,06 \\
\hline \multirow{3}{*}{ mar } & 7 & 0,91 & 0,36 & 1,20 & 0,23 & 1,36 & 0,17 \\
\hline & 8 & 1,16 & 0,25 & 1,33 & 0,18 & 1,42 & 0,16 \\
\hline & 9 & 0,67 & 0,50 & 1,06 & 0,29 & 1,24 & 0,21 \\
\hline \multirow{3}{*}{$a b r$} & 10 & 0,79 & 0,43 & 1,45 & 0,15 & 1,92 & 0,06 (1983) \\
\hline & 11 & 0,77 & 0,44 & 1,27 & 0,20 & 1,64 & $0,10(1971)$ \\
\hline & 12 & 0,92 & 0,36 & 1,47 & 0,14 & 2,05 & $0,04(1978)$ \\
\hline \multirow{3}{*}{ mai } & 13 & 0,61 & 0,54 & 0,31 & 0,76 & 0,55 & 0,44 \\
\hline & 14 & 0,55 & 0,58 & 0,45 & 0,65 & 0,35 & 0,75 \\
\hline & 15 & 1,99 & 0,05 (1980) & 1,91 & 0,06 (1967) & 1,85 & 0,06 (1967) \\
\hline \multirow{3}{*}{ jun } & 16 & $-0,16$ & $0,88(1954)$ & $-0,16$ & $0,88(1954)$ & 0,24 & 0,81 (1954) \\
\hline & 17 & $-0,04$ & 0,97 (1959) & 0,39 & 0,70 (1959) & 0,74 & $0,46(1980)$ \\
\hline & 18 & 1,08 & $0,28(1965)$ & 1,08 & $0,28(1965)$ & 1,06 & 0,29 (1965) \\
\hline \multirow{3}{*}{ jul } & 19 & $-0,38$ & 0,70 (1998) & 0,32 & 0,75 (1998) & 0,75 & 0,45 (1980) \\
\hline & 20 & 1,96 & 0,05 (1967) & 1,99 & 0,05 (1967) & 1,76 & $0,08(1967)$ \\
\hline & 21 & 1,26 & $0,21(1968)$ & 1,21 & $0,22(1968)$ & 1,03 & $0,30(1968)$ \\
\hline \multirow{3}{*}{ ago } & 22 & 1,89 & $0,06(1962)$ & 0,99 & 0,16 & 1,30 & 0,18 \\
\hline & 23 & 0,10 & 0,92 & 0,40 & 0,69 & 0,42 & 0,67 \\
\hline & 24 & 0,78 & 0,44 & 0,93 & 0,35 & 1,34 & 0,18 \\
\hline \multirow{3}{*}{ set } & 25 & 0,57 & 0,57 & 0,57 & 0,57 & 1,08 & 0,28 \\
\hline & 26 & 2,38 & $0,02(1981)$ & 2,53 & $0,01(1982)$ & 2,66 & $0,01(1982)$ \\
\hline & 27 & 0,73 & 0,47 & 0,79 & 0,43 & 0,73 & 0,47 \\
\hline \multirow{3}{*}{ out } & 28 & 1,74 & 0,08 (1969) & 1,63 & $0,10(1971)$ & 1,51 & 0,13 \\
\hline & 29 & 0,73 & 0,47 & 0,90 & 0,37 & 0,99 & 0,32 \\
\hline & 30 & 0,28 & 0,78 & 0,64 & 0,52 & 1,06 & 0,29 \\
\hline \multirow{3}{*}{ nov } & 31 & 1,11 & 0,27 & 2,11 & 0,03 (1976) & 2,57 & 0,01 (1976) \\
\hline & 32 & 1,14 & 0,25 & 1,19 & 0,23 & 1,39 & 0,17 \\
\hline & 33 & 1,87 & $0,06(1972)$ & 2,12 & 0,03 (1972) & 2,47 & $0,01(1972)$ \\
\hline \multirow{3}{*}{ dez } & 34 & 0,65 & 0,51 & 1,08 & 0,28 & 1,23 & 0,22 \\
\hline & 35 & 2,28 & $0,02(1978)$ & 2,37 & $0,02(1983)$ & 2,30 & $0,02(1983)$ \\
\hline & 36 & 2,13 & $0,03(1992)$ & 2,25 & $0,02(1992)$ & 2,42 & 0,02 (1992) \\
\hline
\end{tabular}

te adotadas como não significativas por não atingirem o valor arbitrário de 5\% de significância, por exemplo), resultaram em marcantes alteraçóes nas séries de ETR, com o valor p de significância inferior a 0,02 (Tabela 1). Condição oposta a esta pôde também ser verificada no segundo decêndio de junho. Apesar da detecção de elevação na ETP com significância $\mathrm{p}<0,05$ (usualmente aceita como significativa), as séries de ETR, relativas a esse mesmo decêndio, tiveram valores do SMK associados a elevados valores $\mathrm{p}$ de significância $(\mathrm{p}>0,45$; comumente adotados como não significativos).

Essa última característica da análise de alteraçôes climáticas em séries temporais é também descrita pelo relatório da Омм (1975), ao citar que embora a queda da temperatura média de inverno, entre 1910 e a data desse relatório, nas planícies da Inglaterra, ainda não tivesse atingido um nível considerável de significância, o aumento da frequência de nevascas associado a essa queda náo significativa da temperatura, já havia atingido um elevado nível de significância. Observaçôes similares são também encontradas em Yue e Hashino (2003) ao relatar que algumas tendências podem ser analisadas como estatisticamente não significativas embora as mesmas possam ter importantes implicaçōes práticas. Segundo RADZIEJEWSKI e Kundzewicz (2004), a presença de componentes relacionados à mudança nos padrôes climáticos em uma série temporal nem sempre é detectada com satisfatória significância por métodos estatísticos.

As elevações com significância ( $\mathrm{p} \leq 0,05)$, observadas nas séries de ETR (Tabela 1) do segundo decêndio de janeiro e do terceiro decêndio de novembro e dezembro, guardam datas iniciais aproximadamente concomitantes com as tendências positivas observadas nas séries de ETP (início das décadas de 1980, 1974 e 1990 respectivamente). Considerando que esses três meses pertencem à estação úmida da região de Campinas, torna-se natu- 
Tabela 2. Testes Sazonal de Mann-Kendall (SMK) aplicados às séries decendiais (D) da razão entre evapotranspiração real e a potencial, para três valores de capacidade de água disponível (CAD), na localidade de Campinas (SP), de 1948 a 2008. As significâncias (p) associadas ao teste $\mathrm{SMK}$ e o ano de início das tendências climáticas, associadas a $\mathrm{p} \leq 0,10$, são também apresentados

\begin{tabular}{|c|c|c|c|c|c|c|c|}
\hline \multirow{2}{*}{ Mês } & \multirow{2}{*}{ D } & \multicolumn{2}{|c|}{$C A D=50 \mathrm{~mm}$} & \multicolumn{2}{|c|}{$C A D=100 \mathrm{~mm}$} & \multicolumn{2}{|c|}{$C A D=150 \mathrm{~mm}$} \\
\hline & & SMK & p & SMK & p & SMK & p \\
\hline \multirow{3}{*}{ jan } & 1 & 1,50 & 0,13 & 1,49 & 0,14 & 1,47 & 0,14 \\
\hline & 2 & $-1,04$ & 0,30 & $-1,07$ & 0,28 & $-1,05$ & 0,29 \\
\hline & 3 & 1,13 & 0,26 & 1,16 & 0,25 & 1,16 & 0,25 \\
\hline \multirow{3}{*}{ fev } & 4 & $-1,19$ & 0,23 & $-1,18$ & 0,24 & $-1,16$ & 0,25 \\
\hline & 5 & $-0,03$ & 0,98 & $-0,03$ & 0,98 & $-0,03$ & 0,98 \\
\hline & 6 & $-0,43$ & 0,67 & $-0,40$ & 0,69 & $-0,42$ & 0,68 \\
\hline \multirow{3}{*}{ mar } & 7 & 0,24 & 0,81 & 0,22 & 0,82 & 0,21 & 0,84 \\
\hline & 8 & $-0,76$ & 0,45 & $-0,86$ & 0,39 & $-0,90$ & 0,37 \\
\hline & 9 & $-0,62$ & 0,53 & $-0,61$ & 0,54 & $-0,59$ & 0,55 \\
\hline \multirow{3}{*}{$a b r$} & 10 & $-1,65$ & 0,11 & $-1,83$ & 0,07 (1973) & $-1,90$ & $0,06(1973)$ \\
\hline & 11 & $-0,52$ & 0,61 & $-0,68$ & 0,49 & $-0,68$ & 0,49 \\
\hline & 12 & $-1,24$ & 0,22 & $-1,25$ & 0,21 & $-1,34$ & 0,18 \\
\hline \multirow{3}{*}{ mai } & 13 & 0,69 & 0,49 & 0,58 & 0,56 & 0,55 & 0,58 \\
\hline & 14 & 0,17 & 0,87 & 0,13 & 0,90 & 0,12 & 0,91 \\
\hline & 15 & 2,05 & 0,04 (1980) & 1,96 & 0,05 (1975) & 1,87 & 0,06 (1975) \\
\hline \multirow{3}{*}{ jun } & 16 & $-1,09$ & 0,27 & $-1,37$ & 0,17 & $-1,37$ & 0,17 \\
\hline & 17 & $-0,85$ & 0,40 & $-0,73$ & 0,47 & $-0,73$ & 0,47 \\
\hline & 18 & 1,11 & 0,27 & 1,19 & 0,24 & 1,25 & 0,21 \\
\hline \multirow{3}{*}{ jul } & 19 & $-1,58$ & 0,11 & $-1,72$ & 0,09 (1976) & $-1,73$ & 0,08 (1976) \\
\hline & 20 & 1,80 & 0,07 (1966) & 1,58 & 0,11 & 1,54 & 0,12 \\
\hline & 21 & 1,49 & 0,14 & 1,49 & 0,14 & 1,35 & 0,18 \\
\hline \multirow{3}{*}{ ago } & 22 & 0,87 & 0,38 & 0,78 & 0,44 & 0,56 & 0,57 \\
\hline & 23 & 0,02 & 0,98 & 0,17 & 0,86 & 0,16 & 0,87 \\
\hline & 24 & 0,24 & 0,81 & 0,35 & 0,73 & 0,42 & 0,67 \\
\hline \multirow{3}{*}{ set } & 25 & 1,27 & 0,20 & 1,47 & 0,14 & 1,52 & 0,13 \\
\hline & 26 & 1,76 & 0,08 & 1,87 & 0,06 (1975) & 1,85 & 0,06 \\
\hline & 27 & 0,95 & 0,34 & 0,98 & 0,33 & 0,94 & 0,35 \\
\hline \multirow{3}{*}{ out } & 28 & 0,69 & 0,49 & 0,58 & 0,56 & 0,62 & 0,53 \\
\hline & 29 & $-1,17$ & 0,24 & $-1,07$ & 0,29 & $-1,02$ & 0,31 \\
\hline & 30 & $-1,86$ & 0,06 (1983) & $-1,86$ & 0,06 (1983) & $-1,70$ & 0,09 (1968) \\
\hline \multirow{3}{*}{ nov } & 31 & $-1,15$ & 0,25 & $-0,78$ & 0,43 & $-0,56$ & 0,58 \\
\hline & 32 & 0,88 & 0,38 & 0,91 & 0,36 & 0,91 & 0,36 \\
\hline & 33 & 0,46 & 0,64 & 0,61 & 0,54 & 0,75 & 0,45 \\
\hline \multirow{3}{*}{ dez } & 34 & $-1,30$ & 0,19 & $-1,15$ & 0,25 & $-1,13$ & 0,26 \\
\hline & 35 & 0,44 & 0,66 & 0,44 & 0,66 & 0,44 & 0,66 \\
\hline & 36 & 0,97 & 0,33 & 1,03 & 0,30 & 0,99 & 0,32 \\
\hline
\end{tabular}

ral inferir que a elevação da demanda atmosférica, foi o principal modulador dos valores positivos do SMK verificados nas séries de ETR. Infere-se também que esse aumento na ETP tem, de forma geral, sido suprido pela disponibilidade hídrica da localidade sob estudo. Essa última afirmação é corroborada pelas informaçôes ilustradas na tabela 2 que náo evidencia marcantes alteraçóes temporais no regime da ETR/ETP. Para esse quociente agrometeorológico, o único valor significativo do SMK ( $\mathrm{p} \leq 0,05$; terceiro decêndio de maio), parece ter sido fundamentalmente condicionado pela elevação do regime de PRE (Tabela 3).

\section{CONCLUSÃO}

As séries decendiais de evapotranspiração real e da razão entre a evapotranspiração real e potencial da localidade de
Campinas são de persistência temporal, em que, por definição, a probabilidade de ocorrência de um valor de ETR ou ETR/ETP é significativamente condicionada por seus valores precedentes. A elevaçấo nos valores de capacidade de água disponível (CAD) adotadas resultou em aumento dessa persistência. $\mathrm{O}$ uso de métodos matemáticos que assumem independência dos dados da amostra não pode ser recomendado.

Com base nos métodos adotados, não foram observadas relaçóes conclusivas entre a variabilidade temporal dos sinais El Niño/Oscilação Sul e das séries de ETR e ETR/ETP nas escalas intra e interanual. Contudo, na escala interdecadal observou-se possível relação entre as diferentes fases da Oscilação Decadal do Pacífico e a variabilidade temporal desses dois parâmetros agrometeorológicos. Ao longo da fase fria dessa oscilação multidecadal (1948-1976), as anomalias nos sinais temporais da ETR e da ETR/ETP foram mais acentu- 
Tabela 3. Testes Sazonal de Mann-Kendall (SMK) aplicados às séries decendiais (D) de evapotranspiração potencial e precipitaçáo pluvial na localidade de Campinas (SP), de 1948 a 2008. As significâncias (p) associadas ao teste SMK e o ano de início das tendências climáticas, associadas a $\mathrm{p} \leq 0,10$, são também apresentados

\begin{tabular}{|c|c|c|c|c|c|}
\hline \multirow[t]{2}{*}{ Mês } & \multirow[t]{2}{*}{ D } & \multicolumn{2}{|c|}{$\begin{array}{c}\text { Evapotranspiração } \\
\text { potencial }\end{array}$} & \multirow[t]{2}{*}{ SMK } & \multirow{2}{*}{$\begin{array}{c}\text { Precipitação } \\
\text { pluvial } \\
\text { p }\end{array}$} \\
\hline & & SMK & p & & \\
\hline \multirow{3}{*}{ jan } & 1 & $-0,69$ & 0,49 & 1,03 & 0,30 \\
\hline & 2 & 2,23 & 0,03 (1983) & $-0,75$ & 0,45 \\
\hline & 3 & 0,74 & 0,46 & 1,60 & 0,10 (1970) \\
\hline \multirow{3}{*}{ fev } & 4 & 1,66 & $0,10(1968)$ & $-0,58$ & 0,56 \\
\hline & 5 & 0,16 & 0,88 & 0,19 & 0,85 \\
\hline & 6 & 2,12 & 0,03 (1972) & $-0,75$ & 0,46 \\
\hline \multirow{3}{*}{ mar } & 7 & 2,56 & 0,01 (1997) & 0,19 & 0,85 \\
\hline & 8 & 1,34 & 0,18 & $-0,07$ & 0,95 \\
\hline & 9 & 2,16 & 0,03 (1966) & 0,01 & 0,99 \\
\hline \multirow{3}{*}{$a b r$} & 10 & 2,96 & $0,01(1984)$ & $-0,61$ & 0,54 \\
\hline & 11 & 2,70 & $0,01(1976)$ & 1,13 & 0,26 \\
\hline & 12 & 3,90 & 0,01 (1979) & 0,04 & 0,97 \\
\hline \multirow{3}{*}{ mai } & 13 & 1,16 & 0,24 & 1,75 & $0,08(1983)$ \\
\hline & 14 & 0,09 & 0,93 & 0,35 & 0,73 \\
\hline & 15 & 0,44 & 0,66 & 2,45 & $0,02(1976)$ \\
\hline \multirow{3}{*}{ jun } & 16 & 1,50 & 0,13 & $-1,29$ & 0,20 \\
\hline & 17 & 2,02 & 0,04 (1993) & $-1,24$ & 0,21 \\
\hline & 18 & 0,88 & 0,38 & 1,75 & $0,08(1985)$ \\
\hline \multirow{3}{*}{ jul } & 19 & 2,75 & $0,01(1976)$ & $-1,45$ & 0,15 \\
\hline & 20 & 0,17 & 0,87 & 1,75 & 0,08 (1998) \\
\hline & 21 & 0,45 & 0,65 & 1,38 & 0,17 \\
\hline \multirow{3}{*}{ ago } & 22 & 1,30 & 0,19 & 0,27 & 0,79 \\
\hline & 23 & 0,14 & 0,89 & $-0,90$ & 0,37 \\
\hline & 24 & 2,37 & $0,02(1993)$ & 0,13 & 0,90 \\
\hline \multirow{3}{*}{ set } & 25 & 0,13 & 0,90 & 0,45 & 0,65 \\
\hline & 26 & 0,05 & 0,96 & 1,80 & 0,07 (1974) \\
\hline & 27 & $-0,85$ & 0,39 & 0,55 & 0,58 \\
\hline \multirow{3}{*}{ out } & 28 & 0,63 & 0,53 & 1,47 & 0,14 \\
\hline & 29 & 2,64 & 0,01 (1989) & $-1,39$ & 0,16 \\
\hline & 30 & 2,59 & $0,01(1976)$ & $-1,37$ & 0,17 \\
\hline \multirow{3}{*}{ nov } & 31 & 1,91 & $0,06(1976)$ & 0,07 & 0,94 \\
\hline & 32 & 1,69 & $0,09(1984)$ & 1,04 & 0,30 \\
\hline & 33 & 2,13 & $0,03(1976)$ & 0,44 & 0,66 \\
\hline \multirow{3}{*}{ dez } & 34 & 2,50 & $0,01(1985)$ & $-0,65$ & 0,51 \\
\hline & 35 & 1,82 & 0,07 (1984) & 0,81 & 0,42 \\
\hline & 36 & 2,02 & 0,04 (1993) & 0,82 & 0,41 \\
\hline
\end{tabular}

adas quando comparadas às observadas durante a fase quente (subsequente) dessa oscilação da temperatura do Oceano Pacífico.

Dentre os dois parâmetros agrometeorológicos analisados, as séries decendiais de ETR estão mais sujeitas às tendências de elevação em seus dados. A razão ETR/ ETP foi pouco afetada pelas alteraçôes climáticas observadas nas séries de precipitação pluvial e, especialmente, da evapotranspiraçấo potencial. Considerando os métodos adotados, conclui-se que o suprimento hídrico característico da região de Campinas, representado pelos totais decendiais de precipitação pluvial, foi capaz de suprir essa elevação da demanda hídrica atmosférica observada entre 1948 e 2008.

\section{AGRADECIMENTO}

Os autores agradecem ao Dr. Aslak Grinsted pelo algoritmo para análise cruzada de ondaletas e ao revisor anônimo pelas sugestôes relativas à análise espectral de ondaletas.

\section{REFERÊNCIAS}

ALLEN, R.G.; PEREIRA, L.S.; RAES, D.; SMITH. M. Crop evapotranspiration: guidelines for computing crop water requirements. Roma: FAO, 1998. 300p. (Irrigation and Drainage, Paper 56)

ANDREOLI, R.V.; KAYANO, M.T. ENSO-related rainfall anomalies in South America and associated circulation features during warm and cold Pacific decadal oscillation regimes. International Journal of Climatology, v.25, p. 2071-2030, 2005.

BLAIN, G. C. Consideraçóes estatísticas relativas à oito séries de precipitaçáo pluvial da Secretaria de Agricultura e Abastecimento do Estado de São Paulo. Revista Brasileira de Meteorologia, v.24, p.12-23, 2009.

BLAIN, G. C.; ARAUJO, M. C.; LULU, J. Análises estatísticas das tendências de elevação nas séries anuais de temperatura mínima do ar no Estado de São Paulo. Bragantia, v.68, p.807-815, 2009a.

BLAIN, G. C.; KAYANO, M. T.; CAMARGO, M. B. P.; LULU, J. Variabilidade amostral das séries mensais de precipitação pluvial em duas regiôes do Brasil: Pelotas-RS e Campinas-SP. Revista Brasileira de Meteorologia, v.24, p.1-11, 2009 b.

BLAIN, G. C.; KAYANO, M. T.; SENTELHAS, P. C.; LULU, J. Variabilidade sazonal da evapotranspiraçáo relativa em Campinas (SP): caracterização climática e análise de tendências. Bragantia, v.68, p.545-558, 2009c.

CAMARGO, M.B.P.; ROLIM, G.S.; SANTOS, M.A. Modelagem agroclimática do café: estimativa e mapeamento das produtividades. Informe Agropecuário, v.28, p.58-65, 2007.

CAMARGO, M.B.P., HUBBARD, K.G. Drought sensitivity indices for a sorghum crop. Journal of Production Agriculture, v.12, p.312-316, 1999

DOORENBOS, J.; KASSAM, A.H. Efeito da água no rendimento das culturas. Campina Grande: UFPB, 1994. 306p. (Estudos FAO: Irrigação e Drenagem, 33). Tradução de H.R. Gheyi; A.A. de Souza; F.A.V. Damasco; J.F. de Medeiros.

FREI, C.; SCHAR, C. Detection probability of trends in rare events: Theory and application to heavy precipitation in the Alpine region. Journal of Climate, v.14, p.1568-1584, 2000.

GRINSTED, A.; MOORE, J. C.; JEVREJEVA, S. Application of the cross wavelet transform and wavelet coherence to geophysical time series. Nonlinear Processes in Geophysics, v.11, p. 561-566, 2004.

HIRSCH, R. M., SLACK J. R., SMITH R. A. Techniques of trend analysis for monthly water quality data. Water Resources Research, v.18, p. 107-121, 1982. 
HIRSCH, R. M.; SLACK, J. R. A nonparametric trend test for seasonal data with serial dependence. Water Resources Research, v.20, p.727-732, 1984

IPCC. Climate Change 2007: The Physical Science Basis, Contribution of Working Group I to the Fourth Assessment Report of the Intergovernmental Panel on Climate Change. Cambridge: Cambridge University Press, 2007.

KAYANO, M.T.; ANDREOLI, R.V. Relations of South American summer rainfall interannual variations with the Pacific Decadal Oscillation. International Journal of Climatology, v.27, p.531-540, 2007.

KAYANO, M.T.; BLAIN, G.C. Análisis de ondeletas de series mensuales de precipitación, temperaturas máximas y mínimas mensuales en Campinas. In: CONGRESO VENEZOLANO DE AGROMETEOROLOGIA, 4., 2007. Maracay, Anais... Maracay: SVAGRO, 2007. CD-ROM

KENDALL, M. A.; STUART, A. The advanced theory of statistics. 2.ed. Londres: Charles Griffin, 1967. v.2, 690p.

MANN, H.B. Non-parametric tests against trend. Econometrica, v.13. p.245-259, 1945.

MANTUA, N.J.; HARE, S.R.; ZHANG, Y.; WALLACE, J.M.; FRANCIS, R.C. A Pacific interdecadalclimate oscillation with impacts on salmon production. Bulletin of the American Meteorological Society, v.6, p.1069-1079, 1997.

MORETTIN, P,L.; TOLOI, C.M.C. Análises de séries temporais. 2.ed, São Paulo: Edgard Blucher, 2006. 531p.

MORTATTI, J.; BORTOLETTO JUNIOR., M.J.; MILDE, L.C.E.; PROSBST, J. Hidrologias dos rios Tietê e Piracicaba: Séries temporais de vazão e hidrogramas de cheia. Revista de Ciência e Tecnologia, v.12, p.55-67, 2004.

MORAES, A.V.C., CAMARGO, M.B.P., MASCARENHAS, H.A.A., MIRANDA, M. A.C., PEREIRA, J.C.V.A. Teste e análise de modelos agrometeorológicos de estimativa de produtividade para a cultura da soja na regiáo de Ribeirão Preto, SP. Bragantia, v. 57, p.393-406, 1998.

ORGANIZAÇÃO METEOROLÓGICA MUNDIAL. Climatic Change. Geneve: World Meteorological Organization, 1966. 79p. (WMO, 195; TP, 100; Technical note, 79)

ORTOLANI, A.A.; CAMARGO, M.B.P. Influência dos fatores climáticos na produção: Ecofisiologia da Produção Agrícola. Piracicaba: Potafos, 1987. 249 p.

ORTOLANI, A.A..; SETELHAS, P.C.; CAMARGO, M.B.P.; PEZZOPANE, J.E.M., GONÇALVES, P. S., Agrometeorological model for a seasonal ruber tree yield. Indian Journal of Natural Rubber Research, v.11, p.8-14, 1998.

PEREIRA, A.R.; ANGELOCCI, L.R.; SENTELHAS, P.C. Agrometeorologia: fundamentos e aplicaçóes práticas. Guaíba: Agropecuária, 2002. 478p.
PETTITT, A.N. A non-parametric approach to the change-point problem. Applied Statistic, v.28, p.126-135, 1979.

PICCINI, A.G.; CAMARGO, M.B.P.; ORTOLANI, A.A.; FAZUOLI, L. C.; GALLO, P.B. Desenvolvimento e teste de modelos agrometeorológicos para a estimativa de produtividade do cafeeiro. Bragantia, v.58, p.171-178, 1999.

RADZIEJEWSKI, M.; KUNDZEWICZ, Z.W. Detectability of changes in hydrological records. Hydrological Sciences Journal, v.49, p.39-51, 2004.

REBOITA, M.S.; KRUSCHE, N.; PICCOLI, H.C. Climate variability in Rio Grande, RS, Brazil: A quantitative analysis of contributions due to atmospheric systems. Revista Brasileira de Meteorologia, v.21, p.256-270, 2006.

RAO, V.B.; HADA, K. An experiment with linear regression in forecasting of spring rainfall over south Brasil. Atmosfera, v.7, p.83 $-87,1994$.

RICHARDS, G.R. Change in global Temperature: A statistical analysis. Journal of Climate, v.6, p.556-559, 1993.

ROLIM, G.S.; SENTELHAS, P.C.; BARBIERI, V. Planilhas no ambiente EXCEL ${ }^{\mathrm{TM}}$ para os cálculos de balanços hídricos: normal, seqüencial, de cultura e de produtividade real e potencial. Revista Brasileira de Agrometeorologia, v.6, p.133-137, 1998.

THORNTHWAITE, C.W. An appoach toward a rational classification. Geographical Review, v.38, p.55-94, 1948.

THORNTHWAITE, C.W.; MATHER, J.R. The water balance. Centerton: Drexel Institute of Technology-Laboratory of Climatology, 1955. 104p. (Publications in Climatology, v.8, n.1)

TORRENCE, C; COMPO, G.P. A practical guide to wavelet Analysis, Bulletin of American Meteorological Society, v.79, p.6178, 1998

VICTORIA, R.L.; MARTINELLI, L.A.; MORAES, J.M.; BALLESTER, M.V.; KRUSCHE, A.V.; PELLEGRINO, G.P.; ALMEIDA, R.M.B.; RICHEY, J.E. Surface air temperature variations in the Amazon Region and Its borders during this century. Journal of Climate, v.11, p.1105-1110, 1997.

WILKS, D.S. Theoretical probability distributions. In: STATISTICAL methods in the atmospheric sciences. 2.ed. San Diego: Academic Press, 2006, 629p.

ZHANG, Y.; WALLACE, J,M.; BATTISTI, D. ENSO-like interdecadal variability: 1900-93. Journal of Climate, v.5, p.10041020, 1997.

ZHANG, Y.; NORRIS, J.; WALLACE, J.M. Seasonality of largescale atmosphere-ocean interaction over the North Pacific. Journal of Climate, v.11, p.2473-2481, 1998.

YUE, S., HASHINO, M. temperature trends in Japan: 1900-1996. Theoretical and Applied Climatology, v.75, p.15-27, 2003. 